
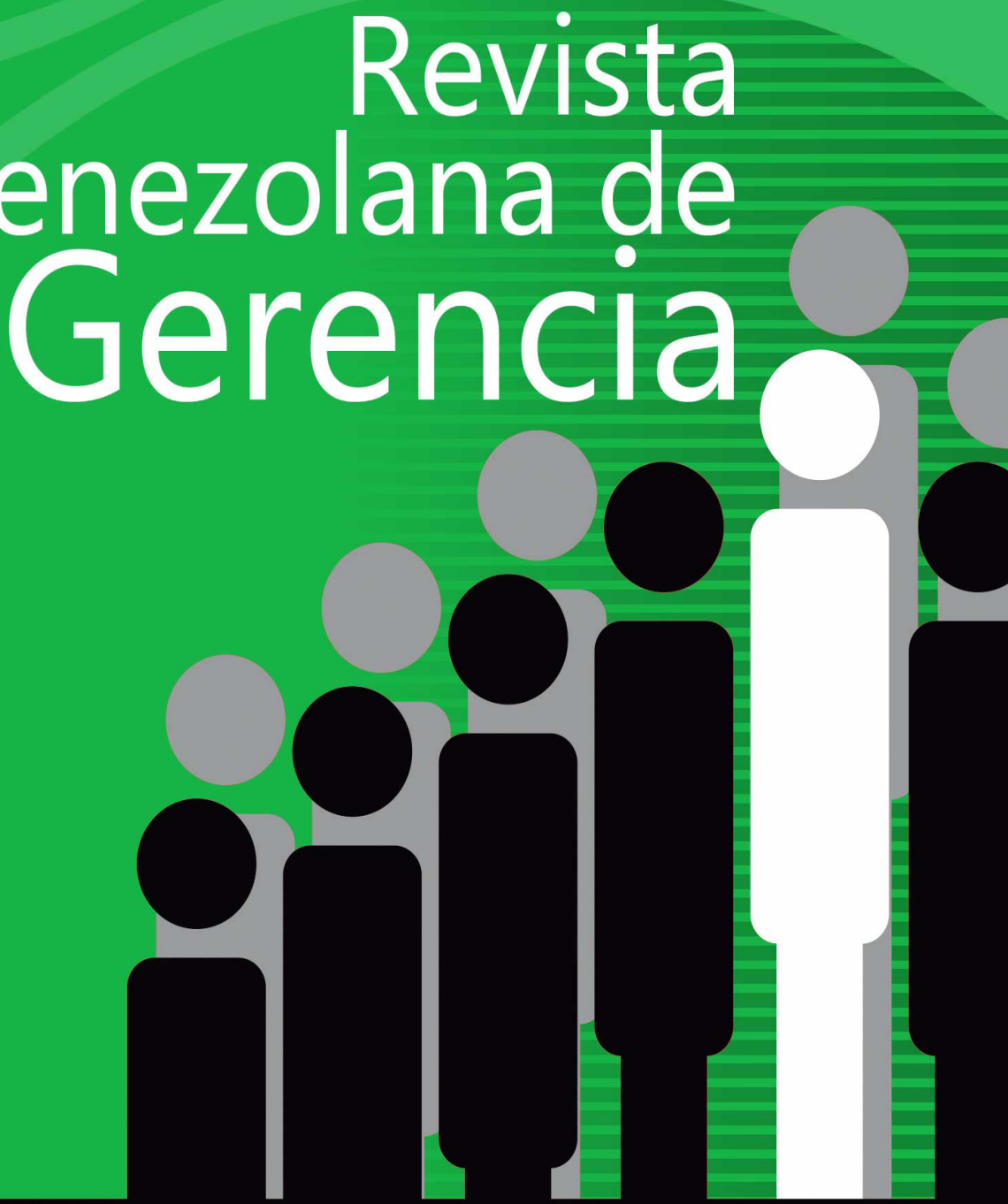


\title{
Centro de competitividad en Colombia: Análisis histórico institucional
}

\author{
Ruano-Ibarra, Elizabeth ${ }^{1}$ \\ Rivera, Wilfred ${ }^{2}$ \\ Alvarado, Oscar ${ }^{3}$
}

\section{Resumen}

En este trabajo, se analizó en perspectiva histórico institucional la trayectoria del Centro Regional de Productividad e Innovación del Cauca. A partir de un abordaje metodológico cualitativo, que priorizó la realización de entrevistas en profundidad y el análisis documental de la normatividad de las políticas de competitividad en Colombia, se correlacionan las directrices normativas con la trayectoria institucional estudiada. Se concluye que las leyes, decretos y documentos Conpes promovidas desde Bogotá fueron determinantes para su creación en el año 2000. Entre las aportaciones del trabajo se destaca la evidencia empírica sobre la pluralidad de dilemas institucionales socioeconómicos y políticos derivados de la implementación de un centro de competitividad como instrumento de las políticas públicas en cuestión.

Palabras clave: competitividad, políticas públicas, Cauca.

Recibido: 11-09-19 Aceptado: 10-11-19

Profesora permanente del Programa de Posgrado en Ciencias Sociales con énfasis en Estudios Comparados sobre las Américas (PPG-ECsA) de la Universidad de Brasilia (UnB). Investigadora del Grupo Interdisciplinar en Ciencias Sociales y Humanas (GIICSH) de la Corporación Autónoma Universitaria del Cauca (Colombia). Correo electrónico: elizabeth.ruano.i@uniautonoma.edu.co, Orcid. https://orcid.org/0000-0003-0549-3951

2 Doctorando en desarrollo regional e integración económica. Docente Corporación universitaria Autónoma del Cauca. Correo electrónico: wilfred.rivera.m@uniautonoma.edu.co. Orcid. https://orcid.org/0000-0003-28887929

3 Magister en Estudios interdisciplinarios del Desarrollo. Director de planeación de la Corporación Universitaria autónoma del Cauca. Correo electrónico: oscar.alvarado.m@uniautonoma.edu.co. Orcid. https://orcid. org/0000-0003-3166-1437 


\title{
Competitiveness center in Colombia: Institutional historical analysis
}

\begin{abstract}
In this work, the trajectory of the Regional Center of Productivity and Innovation of Cauca was analyzed in an institutional historical perspective. Based on a qualitative methodological approach, which prioritized conducting in-depth interviews and documentary analysis of the normativity of competitiveness policies in Colombia, the normative guidelines are correlated with the institutional trajectory studied. It is concluded that CONPES laws, decrees and documents promoted from Bogotá were decisive for its creation in 2000. Among the contributions of the work, it stands out the empirical evidence on the plurality of socio-economic and political institutional dilemmas derived from the implementation of a center for competitiveness as an instrument of the public policies in question.
\end{abstract}

Keywords: competitiveness, public policies, Cauca

\section{Introducción}

Este articulo analiza los resultados del Centro Regional de Productividad e Innovación del Cauca (Crepic) en perspectiva histórico institucional objetivando correlacionar la creación, asociación civil de capital privado y sin ánimo de lucro, constituida en el año 2000 , con la normatividad que direccionó las políticas públicas de competitividad inauguradas en la década de 1990 en Colombia. La investigación realizada en dos etapas ${ }^{4}$, ambas concluidas, indagó sobre la implementación de dichas políticas entre 1992 hasta 2016. Tal delimitación temporal se orientó por la constatación, a partir de la literatura específica, de que el debate sobre competitividad en el país tuvo como hito el informe sectorial de 1992, elaborado por la firma Monitor, en la época presidida por Michel Porter.

Según Reina, Castro y Tamayo (2013), el derrotero político-conceptual que orientó las políticas públicas de incentivo al desarrollo de proyectos exportadores en los años 90 s en Colombia, enfatizó las ventajas regionales y la adopción del enfoque de cadenas productivas y clústeres como

\footnotetext{
4 "Políticas públicas de emprendimiento, innovación y competitividad en el departamento del Cauca: análisis del periodo 1990-2012" y "Nueva institucionalidad de emprendimiento e innovación en el Cauca: Crepic, Creatic y Agroinova", ejecutados entre 2014 a 2016 por el "Grupo Interdisciplinar en Ciencias Sociales y Humanas" (GIICSH) con financiación de la Corporación Universitaria Autónoma del Cauca.
} 
método de priorización para la inversión pública. Lombana y Rozas (2009) y Castro (2013) afirman que esas políticas se han caracterizado por su bajo impacto en las limitaciones estructurales a nivel local. Aunque han sido frecuentes las reestructuraciones al Consejo Nacional de Competitividad (CNC) y al Sistema de Competitividad, principales colegiados nacionales. Sus directrices políticas han carecido de potencia para incidir en los problemas diagnosticados. También sugirió crear centros de innovación y competitividad e inspiró estrategias de dinamización competitiva y priorización local de sectores productivos (Ruano, 2017).

En ese contexto, y a la luz de los indicadores del Banco Mundial (2013 e 2015), que ratificaron el tímido desempeño de la competitividad del Cauca $^{5}$, el Crepic adquirió relevancia como objeto del estudio de caso. Esta institución, en cuanto realidad observada o caso concreto de investigación cuya existencia se materializó al ser proyectado por las políticas en cuestión-, fue asumida como instancia empírica de verificación de los alcances y límites de un instrumento instaurado mediante las políticas y normativas de promoción a la competitividad en Colombia.

Las aportaciones de la investigación y del esfuerzo analítico se hacen relevantes dada la dificultad empírica (costos y tiempo) para rastrear (Aguilar y Lima, 2009) los desenlaces territoriales de las políticas y directrices proyectadas por el poder central gubernamental, localizado en Bogotá capital colombiana. De ese modo, según Olano (2014) y Montecinos (2019) el análisis histórico institucional como perspectiva teóricometodológica contribuye para destacar el surgimiento e institucionalización de decisiones políticas, su evolución e historicidad.

El debruzarse sobre la trayectoria institucional del Crepic, objetiva explicitar las transformaciones alcanzadas, o no, mediante la implementación de las políticas de competitividad. De otro lado, al priorizar la historia institucional para analizar procesos locales se espera contribuir teóricamente al estudio de la competitividad territorial. Para tal finalidad, la trayectoria se establece a partir de los resultados misionales del Crepic contrastándolos con los casos en que la literatura relató trayectorias contrarias.

\section{Consideraciones metodológicas de la investigación}

El análisis documental se realizó en dos etapas y priorizó un total de 26 documentos descritos mediante el cuadro 2 y el cuadro 3 . Dos instancias temporales guiaron el estudio de las fuentes documentales: i) 1992 - 2016 buscando comprender las dinámicas normativas (Cuadro 2) e ii) 2000 - 2016 , década y media de existencia del Crepic (Cuadro 3). La primera etapa analítica estuvo orientada a la identificación del marco regulatorio expedido entre 1992 - 2016 el cual se constató conformado por seis instrumentos jurídico-políticos (Cuadro 1): una ley, dos decretos de cobertura nacional y tres documentos del Consejo Nacional de Política Económica y Social (Conpes), en adelante Conpes.

Vigésimo lugar en infraestructura en un ranking de 29 unidades y Popayán, capital del Cauca, ocupó el decimonoveno lugar entre 23 ciudades capitales en apertura de empresas. 
Cuadro 1.

Cronología y autoría de la normatividad sobre competitividad.

\begin{tabular}{|c|c|c|c|}
\hline Año & & Denominación & Autoría \\
\hline 1994 & 1. & Conpes n. 2739 & Departamento Nacional de Planeación (DNP) \\
\hline 1999 & 2. & Decreto n. 2350 & Presidente de la república \\
\hline \multirow{2}{*}{2004} & 3. & Ley n. 811 & Presidente de la república \\
\hline & 4. & Conpes n. 3297 & DNP \\
\hline 2008 & 5. & Conpes n. 3527 & DNP \\
\hline 2012 & 6. & Decreto n. 1500 & Presidente de la república \\
\hline
\end{tabular}

Fuente: Elaboración propia

La autoría del marco normativo revela la primacía del nivel central tanto el poder legislativo (aprobación de leyes y decretos) como el poder ejecutivo (aprobación de leyes, decretos y Conpes) - en la promulgación de cada instrumento jurídico-político. Desde
1967 los Conpes formalizan decisiones gubernamentales en los diferentes niveles territoriales del país (Melo, 2003), en este caso se vislumbran como instrumento que operacionalizó territorialmente la política pública.

Cuadro 2.

\section{Descripción de las fuentes documentales sobre la normatividad}

\begin{tabular}{|c|c|c|}
\hline Nivel & Denominación & Objetivo/resultado \\
\hline Ley & Ley Nº 811 de 2004 & $\begin{array}{l}\text { Reglamentó el enfoque de cadenas produc- } \\
\text { tivas. }\end{array}$ \\
\hline \multirow{2}{*}{ Decreto } & Decreto $N^{\circ} 2350$ de 1999 & $\begin{array}{l}\text { Creó los Comités Asesores Regionales de } \\
\text { Comercio Exterior (Carce). }\end{array}$ \\
\hline & Decreto $N^{\circ} 1500$ de 2012 & $\begin{array}{l}\text { Actualizó el Sistema Nacional de Competitivi- } \\
\text { dad e Innovación. }\end{array}$ \\
\hline \multirow{3}{*}{ Conpes } & Conpes Nº 2739 de 1994 & $\begin{array}{l}\text { Creó los Centros Regionales de Competitivi- } \\
\text { dad (CRP). }\end{array}$ \\
\hline & Conpes N 3297 de 2004 & $\begin{array}{l}\text { Creó la agenda interna para la productividad y } \\
\text { la competitividad. }\end{array}$ \\
\hline & Conpes $N^{\circ} 3527$ de 2008 & Definió la política nacional de competitividad. \\
\hline
\end{tabular}

Fuente: Elaboración propia 
La secuencia de cada instrumento normativo contribuyó legitimando territorial, jurídica y políticamente los lineamientos de la política de competitividad. Es decir, la normatividad permitió crear instancias de articulación público-privadas, como los CRP y Carce, responsables por la promoción de la competitividad en el ámbito local (Ruano, 2017). La trayectoria del Crepic inició seis años después de la promulgación del Conpes n. 2739 de 1994 el cual amparó la creación los CRP.
Esos cotejos aparentemente obvios, se lograron cruzado fuentes de carácter local (entrevistas y Cuadro 3 ) y nacional (Cuadro 2).

La segunda etapa del análisis documental se enfocó en la consulta de 20 documentos de autoría del Crepic, entre ellos el acta de constitución (2000), tres artículos publicados en la web institucional y 15 informes anuales de gestión del período 2000 al 2015, conforme síntesis a seguir.

\section{Cuadro 3}

Fuentes documentales sobre el Crepic.

\begin{tabular}{lllll}
\hline Denominación & Año & Tipo & $\begin{array}{l}\text { Categorías } \\
\text { (Cuadro 6) }\end{array}$ & Tamaño \\
\hline Acta de constitución. & 2000 & Acta & 1 y 2 & 2 pp. \\
\hline Misión, visión, principios y políticas. & 2015 & Artículo & 1 y 2 & 1 pp. \\
\hline Asociatividad Empresarial. & 2016 & Artículo & 4 & - \\
\hline CREPIC 15 Años. & 2015 & Video & 2 y 3 & $10: 45 \mathrm{~min}$ \\
\hline CREPIC. & 2012 & Artículo & 1,2 y 4 & $11 \mathrm{pp}$. \\
\hline 15 informes de gestión. & $2000-2015$ & Informe & $1,2,3$ y 4 & $721 \mathrm{pp}$. \\
\hline
\end{tabular}

Fuente: Elaboración propia

Dado el énfasis interpretativo del institucionalismo histórico el Cuadro 4 presenta las cuatro categorías - (1) concepción y evolución; (2) modelo de gestión; (3) organización jurídica y (4) resultados y su respectivo objetivo analítico - que emergieron con la investigación. De ese modo, se tensionó la mirada resultadista que mide la trayectoria institucional apenas a partir de resultados cuantitativos. Así, el análisis histórico institucional es eficaz para correlacionar la trayectoria del Crepic con las directrices de competitividad inauguradas en los años 90 porque, según Olano (2014), y Valdéz (2019) se reconoce tanto la reciprocidad entre la acción social y el entorno político y socioeconómico como la capacidad del Estado - actor principal - para imponer decisiones o controlar las reglas unidireccionalmente. 


\section{Cuadro 4}

Categorías del análisis documental sobre la trayectoria del Crepic.

\begin{tabular}{ll}
\hline \multicolumn{1}{c}{ Categoría } & \multicolumn{1}{c}{ Objetivo analítico } \\
\hline Concepción y evolución. & $\begin{array}{l}\text { Correlacionar dinámicas externas (entorno nacional, regional y } \\
\text { local) y actores que contribuyeron con su promoción y desempeño. } \\
\text { Demarcar hitos institucionales. }\end{array}$ \\
\hline Modelo de Gestión. & $\begin{array}{l}\text { Comparar el modelo de gestión (organigrama, planta de personal, } \\
\text { perfiles profesionales, formas de contratación, procesos financieros } \\
\text { y operacionales) inicial (2000) y final (2015). }\end{array}$ \\
\hline Organización jurídica. & Analizar los cambios en la constitución jurídico-institucional. \\
\hline Resultados. & Mapear los proyectos ejecutados y el público objetivo. \\
\hline
\end{tabular}

Fuente: Elaboración propia

La realizaron de cinco entrevistas con actores clave en la trayectoria institucional del Crepic se fundamentó en el presupuesto sobre la importancia de la información cualitativa para la comprensión institucional de un caso con alcance temporal prolongado. La experiencia - vínculo institucional superior a un año y desempeño en la toma de decisiones - de los actores entrevistados contribuyó revelando aspectos ignorados por los documentos analizados (Cuadro 5). Ninguna de las personas entrevistadas exigió anonimato puesto que sería ineficaz ya que esos vínculos profesionales se constituyen actualmente como información de 'dominio público'.

\section{Cuadro 5}

\section{Perfil de los entrevistados.}

\begin{tabular}{|c|c|c|c|c|}
\hline \multirow[b]{2}{*}{ Nombre } & \multicolumn{4}{|c|}{ Criterios de selección } \\
\hline & $\begin{array}{l}\text { Antigüedad del } \\
\text { vínculo institu- } \\
\text { cional }\end{array}$ & $\begin{array}{c}\text { Naturaleza del } \\
\text { vínculo institu- } \\
\text { cional }\end{array}$ & Accesibilidad & $\begin{array}{c}\text { Disposición para } \\
\text { participar de la } \\
\text { investigación. }\end{array}$ \\
\hline $\begin{array}{l}\text { Adolfo Plazas } \\
\text { Tenorio. }\end{array}$ & $2000-2007$ & Director & Alta & Favorable \\
\hline $\begin{array}{l}\text { Laura López } \\
\text { Castrillón. }\end{array}$ & 2004-2005 & Directora (e) & Alta & Favorable \\
\hline $\begin{array}{l}\text { Eduardo Rojas } \\
\text { Pineda. }\end{array}$ & $2000-2012$ & $\begin{array}{l}\text { Presidente de la } \\
\text { Junta directiva. }\end{array}$ & Alta & Favorable \\
\hline $\begin{array}{l}\text { Rafael Bermú- } \\
\text { dez Ortega }\end{array}$ & 2006-2012 & Colaborador. & Alta & Favorable \\
\hline $\begin{array}{l}\text { Sandra Rebolle- } \\
\text { do Acosta. }\end{array}$ & 2000-actual & Colaborador. & Alta & Favorable \\
\hline
\end{tabular}

Fuente: Elaboración propia 
Se utilizó el software TextStat, de licencia libre, para analizar la frecuencia de enunciación de las categorías analíticas y el contexto discursivo tanto del contenido documental como de las narrativas de las entrevistas. El análisis contextual buscó fundamentar las inferencias en función de las categorías de estudio antes citadas.

\section{Centros de competitividad como instrumento de política pública en Colombia: discusión.}

Por definición los CRP en Colombia objetivan la "promoción de la productividad y la competitividad para la mejora de la calidad de vida" (Palacios Preciado y Duque Oliva, 2011: 31). Para tal finalidad la normatividad los constituyó como articuladores científicos y tecnológicos en el ámbito local (Colombia, 2008; López, y Torres 2018). En ese sentido, el principal aporte de esta investigación que versa sobre un estudio de caso de un centro de competitividad radica en ofrecer un panorama descriptivo de las dimensiones constitutivas de la trayectoria institucional de los CRP. Ese esfuerzo sistematizador y analítico de los CRP como objeto de estudio aún es raro en el debate académico en los países de este continente.

De acuerdo con las y los entrevistados, el Crepic fue creado en el ámbito de un proyecto de investigación que fue ejecutado por la Universidad de Cauca y financiado por el Departamento Administrativo de Ciencia, Tecnología e Innovación (Colciencias). Esa constatación es importante para redimensionar los alcances de las políticas públicas, las cuales impactan la configuración del entorno (Velásquez, 2009) y afectan el comportamiento de las personas y de las organizaciones. Es decir, a través de ellas los gobiernos determinan normas, procedimientos y prioridades, fijan cursos de acción (Aguilar \& Lima, 2009) y afectan significativamente el presente y futuro social.

Según las entrevistas, desde 1960 - tres décadas antes del inicio del debate gubernamental sobre competitividad en Colombia - la Universidad del Cauca había liderado iniciativas de articulación entre el sector productivo y la institucionalidad de apoyo, como sintetiza el cuadro 6. En la memoria de las(los) entrevistadas(os), la articulación interinstitucional agenciada por la universidad pública local constituyó espacios de aprendizaje y experiencias fundamentales para la creación del Crepic. Desde la década de 1960, conforme la información del siguiente cuadro, la Universidad del Cauca en alianza con otras instituciones había alcanzado importantes conocimientos que serían potenciados mediante el debate sobre competitividad.

Los cuadros 2 y 3 mostraron que la creación de los CRP en Colombia y el Crepic específicamente fue demarcada por ese marco regulatorio sobre competitividad. El primer instrumento gubernamental sobre competitividad, denominado Conpes número 2739 de 1994, objetivó apalancar la promoción de la competitividad (Melo, 2003) en el contexto conocido como la apertura económica (Cabrera, López, \& Ramírez, 2011; Lombana \& Rozas, 2009). La ruta de la competitividad (Burbano, González, \& Moreno, 2011) y la formulación de la política nacional en la década de 2000 (Castro, 2013) retomaron las 


\section{Cuadro 6}

\section{Antecedentes de articulación institucional en el Cauca.}

\begin{tabular}{|c|c|c|}
\hline Año & & Factor relevante \\
\hline 1960 & \multicolumn{2}{|c|}{$\begin{array}{l}\text { Creación de la Facultad de Electrónica y Telecomunicaciones de la Univer- } \\
\text { sidad del Cauca orientada en articulación con las demandas empresariales } \\
\text { del Cauca y financiamiento de Naciones Unidas (Rojas, entrevista 2016). }\end{array}$} \\
\hline 1970 & \multicolumn{2}{|c|}{$\begin{array}{l}\text { Creación del grupo de investigaciones en Ingeniería Telemática y Telecon- } \\
\text { sulta, primera empresa exitosa creada en dicha facultad (ídem). }\end{array}$} \\
\hline 1990 & \multicolumn{2}{|c|}{$\begin{array}{l}\text { Consolidación del Sistema de Investigación, Innovación, Ciencia y Tecnolo- } \\
\text { gía del Cauca (ídem). }\end{array}$} \\
\hline 1995-1998 & \multicolumn{2}{|c|}{$\begin{array}{l}\text { Realización del proyecto "Misión del Pacifico" y del "Plan de Ciencia y Tec- } \\
\text { nología del Pacífico" (ídem). }\end{array}$} \\
\hline $1997-1998$ & \multicolumn{2}{|c|}{$\begin{array}{l}\text { Creación de la Vicerrectoría de Investigaciones (VRI) y del Sistema de In- } \\
\text { vestigaciones de UniCauca (ídem). }\end{array}$} \\
\hline 1998 & \multicolumn{2}{|c|}{$\begin{array}{l}\text { Capacitación de } 45 \text { representantes de los sectores académico, empresarial } \\
\text { y gubernamental en "Ciencia y Tecnología" (López, entrevista 2016). }\end{array}$} \\
\hline 1999 & \multicolumn{2}{|c|}{$\begin{array}{l}\text { Consenso interinstitucional para crear instituciones de articulación académi- } \\
\text { co-productiva en el Cauca (Rojas, entrevista 2016). }\end{array}$} \\
\hline \multirow[t]{4}{*}{2000} & \multirow[t]{2}{*}{ Marzo } & Colciencias convocó proyectos para la creación de CRP. \\
\hline & & Postulación del proyecto para crear el Crepic. \\
\hline & Septiembre & $\begin{array}{l}\text { Ratificación de la creación del Crepic en el ámbito de la } \\
\text { política de Centros de Productividad del Sistema Nacional } \\
\text { de Innovación (Plazas, entrevista 2014). }\end{array}$ \\
\hline & Diciembre & $\begin{array}{l}\text { Construcción de la visión compartida Cauca- } 2020 \text { y con- } \\
\text { solidación de la mesa de competitividad regional (ídem). }\end{array}$ \\
\hline
\end{tabular}

Fuente: Elaboración propia

recomendaciones del estudio Monitor antes citadas.

La memoria documental institucional posee información parcial para correlacionar la creación del Crepic con la normatividad nacional. Ese dificultoso rastreo informacional se explicaría por la disgregación normativa a lo largo de aproximadamente una década (cuadro 1). En segundo lugar, porque las instituciones locales de surgimiento reciente como la Comisión Regional de Competitividad del Cauca y el Crepic, entre otros citados en el siguiente cuadro, priorizan su sobrevivencia ante esfuerzos orientados a documentar su trayectoria institucional.

El Decreto N. 2350 de 1999 amparó la creación de Comités Asesores Regionales de Comercio Exterior (Carce) en el país. El Carce del Cauca, creado en el año 2001, alcanzó una duración corta, menos de dos años, enfocándose durante su existencia a la promoción del Plan Exportador del Cauca (Cuadro 7). La Comisión Regional de Competitividad del Cauca, fue creada en 2007 y aún subsistía en el año de 2016, con el 
propósito de estructurar el denominado Plan Regional de Competitividad del Cauca. Esa comisión surgió en marco de la Agenda interna para la productividad y la competitividad legitimada por el Conpes N. 3297 de 2004. Tanto el
Carce como la Comisión Regional de Competitividad del Cauca fueron dinámicas promovidas por el Crepic a pesar de su corta existencia institucional en la época.

\section{Implementación de las políticas de competitividad en el Cauca}

\begin{tabular}{l}
\hline Marco regulatorio nacional \\
$\begin{array}{l}\text { Conpes } n^{\circ} 2739 / 1994 . \\
\text { Incentivó la creación de CRP. }\end{array}$ \\
$\begin{array}{l}\text { Decreto } n^{\circ} 2350 / 1999 . \\
\text { Creación de Carce. }\end{array}$ \\
\\
Ley $n^{\circ} 811 / 2004$. \\
Reglamentación del enfoque de cadenas \\
productivas.
\end{tabular}

Conpes n³297/2004.

Agenda interna para la productividad y la competitividad.
Implementación normativa en el Cauca

En el año 2000 se creó el CRP del Cauca, Crepic.

En el 2001 se creó el Carce-Cauca.

El Carce-Cauca lideró la priorización de sectores productivos con potencial exportador.

Adopción de la formulación de proyectos como estrategia de financiación gubernamental y de la cooperación internacional.

Fomipyme financió proyectos de fortalecimiento de las cadenas productivas de miel, flores, panela, chontaduro, piscicultura y software, entre 2004 a 2006.

Creación de los consejos técnicos de las cadenas productivas de panela, chontaduro, y piscicultura.

En 2007 se creó la Comisión Regional de Competitividad del Cauca (CRCC).

Construcción de la Agenda Interna "Cauca compite", un ejercicio de priorización y prospección productiva.

Conpes $n^{\circ} 3527$ de 2008.

Definición de la política nacional de com-

En el 2009 se formuló el Plan Departamental de Competitividad.

Decreto $n^{\circ} 1500$ de 2012.

Actualización del Sistema Nacional de

Competitividad e Innovación.

En el 2015 se creó la Secretaría Departamental de Desarrollo Económico y Competitividad (SEDEC)

Fuente: Elaboración propia

El Conpes N. 2739 de 1994 incentivó la creación de CRP en Colombia estableciendo que deberían "servir como entes articuladores de la voluntad política de la institucionalidad territorial, la oferta y la demanda científica y tecnológica desde las esferas productivas e institucionales 
a nivel regional" (Colombia, 2008: 3). Internacionalmente esos centros cumplen, entre otras, las siguientes atribuciones: a) fomentar cambios de actitud para mejorar el desempeño empresarial; b) promover redes entre instituciones educativas y empresas y, c) asesorar en la formulación y ejecución de políticas para la competitividad (Preciado \& Oliva, 2011). El Banco de Desarrollo de América Latina (CAF, 2006), destacó el carácter estructurante de la sostenibilidad financiera en la consolidación de los CRP, en tal dirección sugiere la combinación de aportes público-privados y/o subsidios anuales o plurianuales con fuentes concursables y contratos de servicios con entidades públicas y privadas como prácticas exitosas de financiamiento.

En el mundo los CRP se ocupan del diagnóstico de los problemas de la productividad de la economía nacional, regional, sectorial y empresarial. Para lograr ese propósito buscan la concertación con diferentes entidades que intervienen en las actividades económicas, establecen sistemas de información, realizan investigación aplicada $y$, por lo general, prestan servicios de consultoría y asesoría empresarial (Arias y Gómez, 1999)

Para Porter (1990) la competitividad empresarial implica lograr ventajas frente a los mejores competidores en escala mundial. Su modelo denominado el diamante de la competitividad propone la apropiación de las condiciones del entorno, entre ellas las políticas gubernamentales y el conocimiento de las universidades, para aumentar las capacidades $y$ potenciar las oportunidades y las ventajas comparativas. Recientemente el BID (2015), retomó la interacción productiva promovida por ese modelo, al demandar políticas públicas favorables macroeconómicamente, capaces de incidir en la disponibilidad de recursos financieros, servicios de infraestructura y capital humano, además de promover la articulación entre las empresas y la investigación universitaria en favor de la competitividad.

Arias y Gómez (1999) y Ramírez y Parra-Peña (2010) afirman que la competitividad es unasunto delas regiones puesto que su operacionalización ocurre en el ámbito micro. En aparente sintonía con ese postulado, el gobierno Gaviria, entre 1990 a 1994, promovió la creación de instancias locales para superar la incipiente institucionalidad regional, entre ellas los CRP. Énfasis similar fue observado en la década de 2000 cuando se promovieron la Comisión Regional de Competitividad, instancia colegiada responsables por dinamizar la Agenda Interna. El siguiente cuadro sintetiza tales dinámicas enfatizando las relaciones entre la normatividad construida en Bogotá y los emprendimientos institucionales del Cauca. Interesa recordar que la creación de los CRP no es una invención colombiana, originarios europeos, fueron ampliamente publicitados por organismos internacionales, principalmente, el CAF.

El Crepic se fundó en el año 2000 con la participación de cuatro actores fundadores: Cámara de Comercio del Cauca, Caja de Compensación Familiar del Cauca, Universidad del Cauca y Gobernación del Cauca (Crepic, 2000). Los dos primeros tienen carácter privado y los restantes son públicos, todos con actuación institucional delimitada al Cauca. Aportaron 226 millones de pesos colombianos, 40 en efectivo y 186 en especie, no se encontraron registros sobre la ausencia de aportes de la Gobernación del Cauca (Tabla 1). 
Tabla 1.

Aportes socios fundadores CREPIC (año 2000).

\begin{tabular}{lllll}
\hline \multirow{2}{*}{ Socio } & Total (\$ Cop.) & Modalidad & & Distribución de los aportes \\
\cline { 3 - 5 } CCC & 100.000 .000 & 30.000 .000 & 70.000 .000 & $\begin{array}{l}\text { Efontaje de herramientas tecnológicas” y } \\
\text { varios. }\end{array}$ \\
\hline UniCauca & 66.000 .000 & 10.000 .000 & 56.000 .000 & Salarios, aportes parafiscales y varios. \\
\hline ComfaCauca & 60.000 .000 & -- & 60.000 .000 & Proyectos afiliados. \\
\hline TOTAL & 226.000 .000 & 40.000 .000 & 186.000 .000 & -- \\
\hline
\end{tabular}

Fuente: Elaboración propia

De la tabla anterior se infere que el estrecho financiamento fue una característica fundadora del Crepic el cual según Ruano, Silva e Rivera (2015) y Ruano (2017) ha sido manifestado por los actores locales en diferentes ejercicios ${ }^{6}$ de prospección territorial. En el capital inicial los aportes en especie representaron $82 \%$, esa fragilidad se fue consolidando como patrón persististe en el periodo estudiado. El cotidiano institucional se nutre de 'acrobacias' financieras, administrativas y políticas para viabilizar el cumplimiento de su misión como "institución interfaz que facilita el dialogo entre la academia y los empresarios" (Rojas, entrevista 2016).

El Crepic buscó consolidarse como una organización "virtual, una institución muy liviana que no consumiera recursos y capaz de atraer financiación" (Rojas, entrevista 2016). Es decir, su fundación surge apoyada en dos supuestos de gestión. De un lado, no generar costos de operación $y$, de otro, desarrollar capacidades institucionales para captar dinero de fondos concursables proyectos. A los CRP colombianos, entre otras funciones, se les atribuyó la formulación, gestión y ejecución de proyectos de apoyo a la formación y consolidación de clústeres y redes empresariales (Colombia, 2008). Esta función, es sin duda, el principal campo de actuación institucional del Crepic.

En 2003 y 2015, Comfacauca y la Universidad del Cauca, renunciaron a su condición de instituciones fundadoras. Esa abdicación ocasionó una crisis de gobernanza y legitimidad (Rebolledo, entrevista 2017) dado el prestigio y la importancia del capital simbólico que esos actores aportaban al Crepic. El CAF (2006) trató sobre la acirrada reyerta entre los CRP, las universidades y empresas privadas que ofertan servicios de gestión de proyectos, consultoría y capacitación en los paises lationoamericanos. En este caso, la disputa por la escasa financiación se configuró como consecuencia duradora y determinante para ese conflicto de intereses entre esos actores locales.

Esa dimisión puede ser comprendida como una paradoja de la política en cuestión. En el año 2000 al

6 Visión Cauca 2032 (2010), Pacto por la Innovación (2011), Plan Departamental de Desarrollo 2012-2015 (2012), Contrato Plan (2013) y CONPES Cauca (2013). 
fundar el Crepic, esos actores compartian la importancia de fortalecer una instancia autónoma para la promoción de la competitividad local (López, entrevista 2016). 15 años despues, el Crepic - formulador, postulante y gestor de proyectos - compite con los actores fundadores en las disputas por accesar la financiación de fondos concursables. Palacios Preciado y Duque Oliva (2011) recomiendan a los CRP estimular la capacidad de adaptación y readaptación de sus objetivos misionales a las transformaciones exógenas y reducir las interacciones negativas.

La estructura organizacional de pequeña escala se habia constituido en ventaja comparativa en relación a los actores fundadores (Rebolledo, entrevista 2017). Esa emergencia de conflitos locales 'nuevos' constrastó con la omisión relativa o no responsabilización de los idealizadores de la política de competitividad en el gobierno central (Rivera, entrevista 2016).

Conforme Bejarano (2013: 28), "[...] la competitividad no es un objetivo de política de corto o mediano plazo, sino la búsqueda de una condición sostenible caracterizada por su permanencia". En ese contexto, se esperaría que los agentes gubernamentales que promovieron la creación de los CRP en Colombia contribuyan con estrategias de enfrentamiento de las disfuncionalidades financieras y político-institucionales resultantes del proceso de consolidación del Crepic como instrumento de política pública. En este punto se coincide con Aguiar e Lima (2009), la evaluación de políticas públicas puede ser potenciada por los gobiernos, en sus diferentes niveles, cuando extrapolan los aprendizajes de éxito y de fracaso. El abordaje de las políticas públicas como ciclos que no finalizan en la implementación, sino que requieren de monitoreo y realimentación sistemática aún se presenta como reto.

La trayectoria del Crepic apunta también el encogimiento estatal mediante la implementación de las políticas de competitividad. La decisión gubernamental de proyectar a los CRP colombianos como instituciones autosostenibles financieramente fue negligente de las lecciones aprendidas y difundidas en el ámbito internacional. Esa transferencia de los riesgos de la promoción de la competitividad denota el carácter 'predatório' de la descentralización de la gestión pública.

\section{Trayectoria institucional del Crepic: resultados.}

El Crepic se autodefine como "una institución de apoyo al mejoramiento de la competitividad en el Cauca que busca articular capacidades y conocimiento tecnológico a los procesos de desarrollo social" (Crepic, 2015). Al análizar la escolaridad se observa que la función directiva fue desempeñada mayoritariamente por profesionales con diploma de especialización. Durante los 16 años estudiados, alrededor de ocho fueron conducidos por cuatro profesionales con ese grado instruccional. Dos directores declararon formación de magister y dirigieron la institución por un periodo de tres años y cinco meses. Finalmente, dos directores con formación de pregrado alcanzaron un periodo aproximado de cuatro años, como indica el siguiente cuadro. 
Cuadro 8

Directores CREPIC 2000-2016

\begin{tabular}{clll}
\hline & Nombre & \multicolumn{1}{c}{ Período } & Escolaridad \\
\hline 1. & Adolfo Plazas & Sept. 2000 - Sept. 2002 & Magister \\
\hline 2. & Laura López (e) & Sept. 2002- junio 2003 & Pregrado \\
\hline 3. & Marta Ojeda & Junio 2003- marzo 2005 & Especialista \\
\hline 4. & Rubén Varona & Marzo2005- marzo 2008 & Pregrado \\
\hline 5. & Aura Espinosa & Marzo 2008- Sept. 2013 & Especialista \\
\hline 6. & María Martínez & Sept 2013- Feb 2015 & Magister \\
\hline 7. & Ximena Paz & Feb 2015- Dic 2015 & Especialista \\
\hline 8. & Oriana Mendoza (e) & Ene 2016 - Mar 2017 & Especialista \\
\hline
\end{tabular}

Fuente: Delgado (2017).

La información del cuadro dejar ver que la duración de cada periodo directivo osciló entre uno y dos años, con excepción de los ciclos de 2008 a 2013 que alcanzó cinco años y siete meses y aquel de 2002 a 2003 que fue inferior a un año. Este último se trató de una transición contractual en la cual se optó por la modalidad de encargo hasta la definición de la nueva dirección. La figura del encargo operó mediante atribución de los representantes de las instituciones socias, específicamente de quien se desempeñaba como presidente de la junta directiva del Crepic (Rebolledo, entrevista 2017). Entre los ocho directores del citados, seis fueron mujeres sin embargo, conviene destacar que dos directoras ejercieron el cargo en carácter provisional.

Los periodos de encargo de la dirección del Crepic, apesar de haber ocurrido con baja frecuencia, alcanzaron duración extensa. Em ambos casos, la duración del encargo fue superior a un mes aspecto que llama la atención para la confluencia del cambio y encargo de la dirección y crisis de gobernanza demarcadas por desistencia de las instituciones socias. El primer periodo de encargo duró 10 meses, ocurrió entre septiembre del 2002 a junio del 2003. En ese año Comfacauca renunció a su condición de socia fundadora. De modo similar, el segundo encargo ocurrido entre enero del 2016 a marzo de 2017 , con duración de 15 meses, coincidió con la renuncia de la Universidad del Cauca como socia fundadora del Crepic. Ambos encargos aparecen vinculados al hito configurado por crisis institucionales ocasionadas por la desistencia de dos de los tres socios fundadores.

La fragilidad financiera enfrentada por el Crepic desde su fundación remite a la retracción estatal operacionalizada mediante la implementación de políticas públicas neoliberales como las de competitividad en Colombia. La vulnerabilidad técnica, política y financiera del Crepic debe ser ecuacionada como efecto del marco regulatorio nacional que incidió directamente en su creación y en la legitimidad de dichas políticas en el ámbito local colombiano. En ese sentido, la creación, vida y declinio de 
CRP en el país se compreenden como dinamicas inseridas en el complejo ciclo de la política de competitividad. Por tanto, no sólo es una responsabilidad de los actores locales mas también de los idealizadores en Bogotá.

Al analizar la composición de los equipo de trabajo del año inicial - 2000 y del año final de nuestro recorte temporal - 2016 - se confirma el carácter modesto de las pretensiones organizativas al momento de la fundación del Crepic. Las actividades misionales fueron asumidas inicialmene por seis personas, entre las cuales tres no poseian título universitário. El número de profesionales, el nivel de escolaridad y las modalidades de vinculación demuestran la vulnerabilidad institucional y sirven de referencia para ratificar la improvisación administrativa y financiera como trazo constitutivo (Cudro 9).

\section{Cuadro 9}

Personal vinculado al CREPIC: 2000.

\begin{tabular}{lll}
\hline Cargo & Tipo de vinculación & Formación Académica \\
\hline Director ejecutivo & Profesor 'cedido' por Unicauca & Ingeniería de telecomunicaciones \\
\hline Contador & Prestación de servicios & Contaduría pública \\
\hline Revisor Fiscal & & Administración de empresas \\
\hline Coordinadora de proyectos & Contrato laboral & \\
\hline Secretaria & Pasantía no remunerada (2) & Cara \\
\hline
\end{tabular}

Fuente: Elaboración propia

Sin embargo, en el 2016 el Crepic contaba con 81 profesionales vinculados, 45 mujeres y 36 hombres. Los datos empíricos consolidados en el cuadro 10 corroboran la resiliencia institucional configurada en un significativo aumento, cuantitativo y cualitativo, del equipo de trabajo. El 3\% tenía vinculación mediante contrato laboral y el $97 \%$ bajo la modalidad de prestación de servicios. El predominio de ese tipo de vínculo, por su naturaleza jurídica y carácter temporal, creciente en contextos de expansión capitalista y precarización del trabajo, denota incerteza y riesgo de rotatividad del talento humano. Esa alerta es particularmente delicada para la sostenibilidad técnica el Crepic, dado que el portafolio de servicios institucional se fundamenta, principalmente, en las capacidades intelectuales de sus colaboradores (Tabla 2). 


\section{Tabla 2}

Capital Humano del CREPIC (2016)

\begin{tabular}{|c|c|c|c|c|c|c|c|c|}
\hline \multicolumn{5}{|c|}{ Formación Académica } & \multicolumn{2}{|c|}{ Genero } & \multicolumn{2}{|c|}{ Vinculación } \\
\hline $\begin{array}{l}\text { Técnicol } \\
\text { Tecnólogo }\end{array}$ & Pregrado & Especialista & Magister & Doctor & M & $\mathbf{F}$ & Contrato & Prest. Serv. \\
\hline $2 / 5$ & 52 & 11 & 10 & 1 & 36 & 45 & 2 & 79 \\
\hline
\end{tabular}

Fuente: Delgado (2017).

$64,1 \%$ del equipo de trabajo del Crepic en el año 2016 tenía formación universitaria concluida, $13,5 \%$ con especialización, $3 \%$ maestría, $1 \%$ doctorado y $8,6 \%$ nivel técnico y tecnológico. En cuanto a la experiencia $71,6 \%$ poseía entre 1 y 3 años, $10 \%$ de 4 a 6 años; $13,5 \%$ de 7 a 10 años y 3, $7 \%$ más de una década de experiencia. Esos datos revelan que la misión institucional en ese año era ejercida mayoritariamente por un capital humano con formación universitaria y experiencia de 3 años (Tabla 3). Ese perfil profesional se encuadra en un segmento del mercado de trabajo con demanda significativamente alta y oferta media. No obstante, dicha amenaza puede verse neutralizada por las bajas tasas de empleo características de Popayán, ciudad sede del Crepic.

Tabla 3.

Experiencia laboral equipo de trabajo del CREPIC (2016)

\begin{tabular}{lcccc}
\hline Descripción & \multicolumn{5}{c}{ Edad-años } \\
\cline { 2 - 5 } & $\mathbf{1 - 3}$ & $\mathbf{4 - 6}$ & $\mathbf{7 - 1 0}$ & Mas de 10 \\
\hline Total & 59 & 8 & 11 & 3 \\
\hline
\end{tabular}

Fuente: Delgado (2017).

Esa oportunidad de retener - dismunir la rotatividad de los colaboradores, capital fundamental en el cumplimiento misional del Crepic enseña que su consolidación institucional no es unidireccional y tampoco delimitada geográficamente.

Según los informes de gestión entre 2000 a 2015 su acción instituciona cobijó a sectores productivos de 23 municipios $^{7}$ de nueve departamentos - Amazonas, Caquetá, Guaviare, Vaupés, Guainía,

Silvia, Rosas, Totoró, Morales, Suarez, Bolívar, Puracé, Yumbo, El Tambo, Timbío, Popayán, Santander de Quilichao, Toribio, Almaguer, Cali, Pasto, Mocoa, Villa Garzón, Puerto Leguizamón, Colón, Santiago, Puerto Guzmán y Puerto Caicedo. 
Valle del Cauca, Cundinamarca, Choco, Antioquia y Atlántico. Sin embargo, los reportes de los proyectos ejecutados muestran que el público objetivo se restringió a tres departamentos - Cauca,
Nariño y Putumayo. El trazado del perfil de los 484 sujetos colectivos que conformaron ese público se sistematizó en el Cuadro 10.

Cuadro 10

\section{Público objetivo de los proyectos ejecutados entre 2000 a 2015}

\begin{tabular}{lcl}
\hline Descripción & Cantidad & Localización departamental \\
\hline Resguardos $^{1}$ indígenas & 17 & Cauca. \\
\hline Grupos de investigación & 22 & Cauca y Putumayo. \\
\hline Asociaciones de productores & 100 & Cauca y Putumayo. \\
\hline Empresas & 160 & Cauca, Nariño y Putumayo. \\
\hline Instituciones educativas & 185 & Cauca, Putumayo y Nariño. \\
\hline Total & 484 & Cauca, Nariño y Putumayo \\
\hline
\end{tabular}

${ }^{8}$ Instituciones jurídicas y sociopolíticas con carácter étnico localizadas en territorios cuya propiedad es colectiva e inalienable. Se gobiernan por un estatuto especial relativamente autónomo.

Fuente: Delgado (2017).

La perspectiva historicoinstitucional permite observar el despliegue de procesos políticos en el tiempo y su encaje mediante reglas formales, rutinas y convenciones legitimadas por la estructura organizacional (Olano, 2014). La prestación de cuentas constituye un momento crítico, marcado por secuencias y rigideces que dificultan que el Crepic - tomadores de decisiones - escapen de la rutas establecida por la política de competitividad. La interacción institucional con sujetos colectivos como asociaciones de productores o empresas encubre la invisibilidad y marginación de la pluralidad social como estrategia de la gubernamentalidad neoliberal. Al reconocerlos como actores de la competitivad local les transfiere los costos y riesgos de la política y simultaneamente posiciona ciertas modalidades de representación jurídica y social en detrimento de otras.

Las pecualiaridades analizadas anteriormente se traducen en prácticas no ortodoxas a la luz del debate clásico sobre competitividad empresarial. Se constata que las empresas, principal objetivo de la política de competitividad colombiana, representan un modesto $33 \%$ del total establecido como público objetivo. Las insitituciones educativas representan $38 \%$, la suma de los reguardos indígenas y las asociaciones de productores $24 \%$ y los grupos de investigación 4,5\%. Esas informaciones son relevantes para retomar la pregunta sobre el tipo de competividad promovida por el Crepic. Una primera inferencia apunta que aproximadamente $57 \%$ del público objetivo podría encuadrarse como 'empresarial', puesto que los proyectos con las asociaciones y los 
resguardos indígenas objetivaron el mejoramiento productivo para aceder mercados.

Sin embargo, en la plataforma estrategica del Crepic se afirma "tejemos redes de confianza y articulamos sueños. Nos esforzamos por asociar la solidaridad y la colaboración" (Crepic, 2016). Esos términos llaman la atención por su distanciamiento con los presupuestos de la competitividad empresarial. Ese discurso del Crepic sugiere una aproximación a las prácticas políticas emancipadoras indígenas que resisten en el Cauca. Los datos empiricos disponíbles no habilitan mayores inferencias, sin embargo esas constaciones preliminares se colocan como provocaciones con potencial teorico para ser abordadas a futuro.

El Crepic a pesar de estar constantemente amenazado por la vulnerabilidad financiera ya referida simultaneamente promovió su misión, no solo en las fronteras departamentales, y alcanzó su sobrevivencia institucional. Se consolidó como institución gestora de proyectos para el fomento de cadenas y sectores productivos rurales. Enelperiodo 2000-2016 sus capacidades estuvieron direccionadas, principalmente, para la formulación, gestión de financiación y ejecución de un total de 76 proyectos. Esas propuestas obtuvieron financiación de 21 instituciones que en su mayoria administran recursos públicos operados mediante fondos concursables.

Contrastando con las visiones relativistas conviene destacar que los fondos concursables con recursos públicos promueven lógicas excluyentes al ratificar los paradigmas de la escasez de recursos y de la primacia del más apto, entre otros. En esas disputas acirradas y desiguales solamente obtienen financiación estatal los proyectos que se adecuan a las exigencias de cada convocatoria. No basta conocer el campo del proyecto, el dominio de las lógicas de cada financiador es igualmente relevante. No se se obtuvo información para estimar el riesgo institucional asumido por el Crepic en esos procesos y tampoco para comparar el total de proyectos formulados con aquellos financiados (Tabla 4).

Tabla 4

Fuentes de financiación de proyectos (2000-2016).

\begin{tabular}{ccccc}
\hline \multirow{2}{*}{$\mathrm{N}^{\circ}$} & \multicolumn{2}{c}{ Fuentes de financiación } & Total & Monto $^{2}$ \\
\cline { 2 - 4 } Institución & Nivel territorial & Nacional & 18 & $3.330,21$ \\
\hline Colciencias & Nacional & 9 & $1.283,75$ \\
\hline Fomipyme & Municipal & 7 & 269,17 \\
\hline Univ. Georgetown & Internacional & 4 & 168,39 \\
\hline FundaColombia & Internacional & 4 & $1.326,77$ \\
\hline Ministerio Agricultura & Nacional & 4 & 968,02 \\
\hline Gobernación Cauca & Departamental & 3 & $2.177,88$ \\
\hline Gobernación Putumayo & Departamental & 3 & $9.116,50$ \\
\hline
\end{tabular}




\section{Cont... Tabla 4}

\begin{tabular}{ccccc}
\hline \multirow{2}{*}{$\mathrm{N}^{\circ}$ Fuentes de financiación } & \multirow{2}{*}{ Total } & \multirow{2}{*}{ Monto $^{2}$} \\
\cline { 2 - 3 } Institución & Nivel territorial & & 458,34 \\
\hline Banco Mundial & Internacional & 2 & 722,90 \\
\hline Incoder & Nacional & 2 & $1.312,92$ \\
\hline ADAM & Internacional & 2 & 146,97 \\
\hline $\begin{array}{c}\text { Univ. Politécnica Ca- } \\
\text { taluña }\end{array}$ & Internacional & 2 & Sin datos $^{3}$ \\
\hline Crepic & Departamental & 2 & 490,00 \\
\hline $\begin{array}{c}\text { Banco Inter. de Desar- } \\
\text { rollo }\end{array}$ & Internacional & 1 & 171,00 \\
\hline Univ. Politécnica Madrid & Internacional & 1 & 94,35 \\
\hline DNP & Nacional & 1 & 35,34 \\
\hline Incuagro & Departamental & 1 & 962,70 \\
\hline Universidad del Cauca & Departamental & 1 & 121,00 \\
\hline Alcaldía Miranda & Municipal & 1 & 80,00 \\
\hline Alcaldía Silvia & Municipal & 1 & 32,00 \\
\hline Alcaldía Totoró & Municipal & 1 & 40,00 \\
\hline Alcaldía Rosas & Municipal & 1 & $23.708,92$ \\
\hline TOTAL & & 76 & \\
\hline & & 1 & 1 \\
\hline
\end{tabular}

${ }^{9}$ Millones de pesos colombianos (COP). Cifras aproximativas basadas en información no contable, pues no conseguimos acceder a los libros contables del Crepic.

${ }^{10}$ No encontramos información escrita sobre el monto de esos proyectos. Bolaños (entrevista 2017), manifestó que ambos proyectos se desarrollaron en la modalidad de pasantía estudiantil.

Fuente: Delgado (2017).

La tabla anterior muestra el total de proyectos en los cuales el Crepic participó en una o varias etapas formulación, gestión de la finananciación y ejecución. El contraste del nivel territorial del financiador con el monto muestra que $27 \%$ de los recursos fue de origen internacional; nacional y municipal cada uno con $22 \%$; departamental $18 \%$ y $4 \%$ autofinanciación. Focalizando el total de proyectos y el monto se confirman los niveles nacional e internacional como más relevantes. Este dato ratifica la carencia de recursos financieros del Cauca y sus municipios y revela también capacidades, que en algunos 
casos, se tornaron contrapartidas locales corrientemente exigidas por los financiadores ${ }^{11}$.

La acción del Crepic en escala regional le permitió obtener importantes reconocimientos a su desempeño institucional. Se refieren los tres galadornes, el primero recibido el año 2011, cuando le fue ortorgado el Premio Ventures, como retribución a las ideas de alto impacto económico, social y ambiental. Ese premio fue ofrecido en alianza entre empresas, el Ministerio de Industria y Comercio y el SENA. En el año 2012, mediante la resolución $n^{\circ} 01092$ de Colciencias, fue reconocido como Centro de Desarrollo Tecnológico. En el 2015, el gobierno nacional le atribuyó el premio "Regalías bien invertidas" en reconocimiento a la eficiencia y la transparencia en el manejo de los recursos de inversión pública ${ }^{12}$.

Vale la pena retener la atención en la naturaleza de esos premios los cuales corroboran la resiliencia institucional enunciada anteriormente. Los dos primeros son reconocimientos al desempeño y a la pertinencia misional consolidados mediante la gestión de proyectos. Ese perfil institucional y el conocimiento acumulado es evidenciado en el tercer premio. Investigaciones recientes (Ruano, Silva, \& Rivera, 2015) también le reconocen su lugar como productor de conocimiento sobre encadenamientos productivos rurales de pequeña escala en varios municipios del
Cauca, Popayán, Totoró, Rosas, Suarez, Morales, Silvia, Miranda, Patía y Rosas

Según Palacios Preciado y Duque Oliva (2011), los modelos de planes de negocios de los CRP sugieren examinar recursos, competencias, actividades y procesos para descubrir relaciones novedosas. Esa arquitectura del portafolio de servicios con base en la disponibilidad y necesidades de recursos permite establecer y mantener interacciones positivas y mecanismos de colaboración con los clientes. Sin embargo, la trayectoria del Crepic advierte que ese contexto relacional se tornó frágil en una dinámica territorial en que los clientes y socios estratégicos se tornaron competidores directos en las disputas por financiación con recursos públicos.

\section{Conclusiones}

La creación de los CRP en Colombia en la década de 1990 se tradujo en varios centros dispersos en el territorio nacional, cada uno preocupado en diseñar elementos diferenciadores para su modelo de gestión visando su sostenibilidad. Esa dinámica requiere ser comprendida como desenlace de una amplia publicidad vehiculada por organismos internacionales, comoelCAF. Orientada por la lógica de transferencia de conocimiento y tecnologías "blandas", desde el norte global hacia países o regiones rezagadas - sur global -, la

11 Por cada dólar solicitado se exige que la institución responsable y el público objetivo aporten en 'especie' entre uno a tres. El uso de infraestructura física, como auditorios y laboratorios, y jornadas de trabajo se constituyeron como aportes de los beneficiarios.

12 El período de la premiación coincide con la renuncia de dos socios fundadores emblemáticos, tal constatación denota un movimiento inversamente proporcional en tanto se afirma en el contexto institucional nacional se contrae en el ámbito local. 
creación de CRP como política originaria de Europa fue replicada en países latinoamericanos. Esa dinámica se enmarca en el poderoso instrumental de la "ingeniería" social que desde los años 70 ha prometido resolver la pobreza y estancamiento del desarrollo, al tiempo en que se afirma como campo de investigación académica y actividad social.

La investigación priorizó el abordaje histórico institucional para analizar la trayectoria del Crepic como proceso local de promoción a la competitividad territorial. Se verificó que los actores gubernamentales responsables por el diseño de esa política transfirieron los costos y riesgos de la implementación a los actores locales. Tal directriz es justificable en la lógica de la descentralización estatal pero también permite inferir la racionalidad limitada del Estado, actor principal en la toma de decisiones en el ciclo de esa política. Tal limitación produjo impactos no previstos por una mensuración errónea del descompás existente entre las expectativas de los formulados de la política en Bogotá, a partir de imaginarios desconectados de los territorios nacionales e íntimamente vinculados a las pautas de organismos internacionales como la CAF, y las capacidades del Cauca para la gestión de la competitividad territorial.

El análisis de los CRP como instrumento de política pública en Colombia mostró un vínculo fuerte con el concepto de competitividad empresarial. Diferentemente la trayectoria del Crepic sugiere cierta apertura conceptual para la competitividad territorial. Dicha trayectoria es entendida a partir de los proyectos ejecutados durante década y media indican la orientación hacia sujetos colectivos diversos socioeconómicamente como locus privilegiado. El perfil de ese público justifica el énfasis en proyectos de fortalecimiento asociativo para sectores productivos de pequeña escala. Esa perspectiva misional pragmática parece interpelar la centralidad de las empresas, como actor principal de la política colombiana de competitividad y del concepto de competitividad empresarial como sustento teórico.

No obstante, el avance práctico y académico del concepto de competitividad empresarial ese marco conceptual, en dialogo con la apropiación hecha por el campo de las políticas públicas, aún se encuentra en construcción. En la cimentación de ese debate, el territorio es una dimensión fundamental no apenas por su carácter contextual más principalmente por las múltiples influencias que ejerce sobre la institucionalidad que abriga. Si conforme se defiende en este artículo el Crepic promueve la competitividad territorial sus avances y límites no se disocian del Cauca como territorio objeto de su actividad misional.

El perfil socio-productivo esencialmente rural, y en su mayoría, de pequeño porte, la persistencia de los bajos indicadores socioeconómicos, la incipiente sofisticación y agregación de valor denotan características estructurales del Cauca que, además evidencian los límites de las políticas públicas de competitividad y de sus instrumentos si se les proyecta como un rasero. Esas características territoriales constituyen entrabes de difícil resolución para los presupuestos conceptuales de la competitividad empresarial. De otro lado, si bien la naturaleza de las políticas públicas supone el ejercicio de la autoridad mediante reglas legales la trayectoria histórico-institucional 
del Crepic enseña sobre los límites y posibilidades de la racionalidad e influencia del poder gubernamental. Como se mostró en las páginas anteriores no hubo responsabilización paritaria en la promoción de la competitividad entre los agentes estatales y el CRP.

Lasespecificidadessocioeconómicas del Cauca no son apenas fruto de la inercia institucional $o$ incompetencia territorial. Son producidas por fenómenos políticos y económicos estructurales como, entre otros, la concentración de la tierra, la negligencia gubernamental y estatal en sus diferentes esferas, además de fragilidades en infraestructura productiva, financiera, educativa y de conectividad territorial. Esas variables sobre las cuales el debate académico ya ha ofrecido amplio esclarecimiento, por ser barreras a la competitividad empresarial, han sido sistemáticamente relegadas de la agenda de las políticas en cuestión.

De este modo se destaca que las investigaciones a partir de las cuales se produjeron los datos aquí analizados contribuyeron para explorar los contornos no siempre bien intencionados de las políticas públicas. En territorios como el Cauca, marcados por la vulnerabilidad social, esas políticas de fortalecimiento empresarial inciden en cursos de acción institucional que marginan contingentes sociales mayoritarios, los perdedores, y priorizan territorios ganadores. La existencia de un CRP como instrumento hegemónico de la propomoción a la competitividad es también fruto de procesos temporales prolongados calcados en la historicidad de dinámicas locales de articulación institucional.

Se finaliza argumentando que la competitividad empresarial refiere la capacidad de apropiación de conocimiento e implantación de innovaciones a su ciclo productivo sea este de productos o servicios. En dimensión más amplia, la competitividad territorial que buscan promover los CRP se relaciona con la promoción de capacidades específicas de instituciones como empresas, sectorescadenas productivas, asociaciones de productores, resguardos indígenas, instituciones educativas, grupos de investigación, entre otras.

\section{Referencias bibilográficas}

Aguilar, Carlos., Lima, Marco (2009), ¿Qué son y para que sirven las políticas públicas? Contribuciones a las ciencias sociales, 1-15.

Arias, Alberto \& Gómez, Martha (1999), El papel de los centros tecnológicos y el centro de productividad del pacífico en la competitvidad de la industria vallecaucana en Colombia. Heuristica, 9(1), 101-113.

Berdugo, Elber (2014), Competitividad: conceptos y enfoques recientes. Gestión y desarrollo, 7(2), 157182.

Buerbano, Edy., González, Verena., Moreno, Eugenio (2011), La competitividad como elemento esencial para el desarrollo de las regiones. Una mirada al Valle del Cauca. Gestión y desarrollo, 8(1), 51-78.

Banco Mundial (2015), Indicadores de competitividad. Washington, DC.: Consejo privado de competitividad.

Cabrera, María., Lopez, Paula., Rámirez, Claudia (2011), La competitividad empresarial: un marco conceptual para su estudio. Bogotá D.C: Universidad Central.

Colombia (2008), Politica Nacional de Competitividad y Productividad. Bogota: DNP, 20 p.

Consejo Privado de Competitividad (2015), 
Indice Departamental de competitividad 2015. Bogotá.

CREPIC (2000), Acta de constitucion (Numero 001) Popayan : Consultada 21 de Abril de 2016.

CREPIC (2015), Consolidado de Informes Anuales de Gestion. Popayan: Crepic. $70 \mathrm{p}$.

CREPIC (2016), Mision, Vision, Principios y Politicas. www.crepic.org.co/index. php/es/mision-vision-y-principios, recuperado el 26 de Mayo de 2016.

Corporación Andina de Fomento. Nuevas herramientas para la competitividad. Análisis de los Convenios de Competitividad Exportadora. Bogotá: CAF. 2006, p. 56.

Castro, Felipe. (2013), 20 años de políticas de competitividad en Colombia. Bogotá D.C.: Fedesarrollo.

Delgado, Fabián (2017), Competitividad en el Cauca: análisis de la trayectoria institucional del Crepic. Monografía de pregrado. Programa de Administración de empresas. Corporación Universitaria del Cauca. Popayán: 2017, 101 p.

López, Karin., \& Torres, Virginia (2018), La competitividad empresarial y la innovación producida y transferida desde los Centros CONACYT. Memoria V Congreso Red Internacional de Investigadores en Competitividad, 5(1). pp. 1169-1185.

Lombana, Jahir., Rozas, Silvia (2009), Marco analítico de la competitividad: Fundamentos para el estudio de la competitividad regional. Pensamiento y Gestión, 26, 38.

Melo, Alberto. (2003), Colombia: Los problemas de competitividad de un país en conflicto. Washinton: BID.

Montecinos, Egon (2019), Participación ciudadana en la gestión pública: Una revisión sobre el estado actual. Revista Venezolana de Gerencia, 24(86), 342-362

Olano, Aldo. (2014), El institucionalismo histórico comparado. Lecturas sobre América Latina. Opera, 15(15), 5-29.

Palacios Preciado, Mariana; Duque Oliva, Edison Jair (2011), Modelos de negocio propuesta de un marco conceptual para centros de productividad. Administración \& Desarrollo, 39 (53), 23-34.

Ramirez, Juan., Parra-Peña, Rafael (2010), Escalafón de la competitividad en los departamentos de Colombia, 2009. Bogotá D.C.: CEPAL.

Ruano, Elizabeth (2016), Red Regional de Emprendimiento del Cauca: avances y desafios de un instrumento de política pública. Revista ciencias estratégicas, 24(35), 181-197. http:// dx.doi.org/10.18566/rces.v24n35.a11

Ruano, Elizabeth (2017), Políticas Colombianas de Competitividad y Emprendimiento: aproximación comparativa. Revista de Estudos e Pesquisas sobre as Américas, 11(1), 155-177. https://doi.org/10.21057/ REPAM.V11I1.24786

Ruano, Elizabeth., Silva, Valcilon., Rivera, Wilfred (2015), Cadena productiva y capital social: el caso de la piscicultura del Cauca, Colombia. Interações, 16(2), 257-264. https:// doi.org/10.1590/151870122015202

Valdez, Andrés. (2019), Paradigmas emergentes en la gestión pública en América Latina. Revista Venezolana de Gerencia, 24(86), 325-339.

- Esta obra está bajo una licencia de Creative Commons Reconocimiento-NoComercialCompartirlgual 3.0 Unported. http://creativecommons.org/licenses/by-nc-sa/3.0/deed.es_ES 